\title{
Active Signal Conduction through the Sensory Dendrite of a Spider Mechanoreceptor Neuron
}

\author{
Ewald Gingl and Andrew S. French \\ Department of Physiology and Biophysics, Dalhousie University, Halifax, Nova Scotia, B3H 4H7 Canada
}

\begin{abstract}
Rapid responses to sensory stimulation are crucial for survival. This must be especially true for mechanical stimuli containing temporal information, such as vibration. Sensory transduction occurs at the tips of relatively long sensory dendrites in many mechanoreceptors of both vertebrates and invertebrates, but little is known about the electrical properties of these crucial links between transduction and action potential generation. The VS-3 slit-sense organ of the spider Cupiennius salei contains bipolar mechanosensory neurons that allow voltage-clamp recording from the somata, whereas mechanotransduction occurs at the tips of 100 - to 200 - $\mu \mathrm{m}$-long sensory dendrites. We studied the properties of VS-3 sensory dendrites using three approaches. Voltage-jump experiments measured the spread of voltage outward from the soma by observing total mechanically transduced charge recovered at the soma as a function of time after a voltage jump. Frequency-response measurements between pseudorandom mechanical stimulation and somatic membrane potential estimated the passive cable properties of the dendrite for voltage spread in the opposite direction. Both of these sets of data indicated that the dendritic cable would significantly attenuate and retard a passively propagated receptor potential. Finally, current-clamp observations of receptor potentials and action potentials indicated that action potentials normally start at the distal dendrites and propagate regeneratively to the soma, reducing the temporal delay of passive conduction.
\end{abstract}

Key words: mechanoreceptor; sensory transduction; voltage jump; cable; dendrite; propagation; frequency response; excitability

\section{Introduction}

Transduction in many mechanoreceptor neurons of both vertebrates and invertebrates occurs at the ends of fine dendritic processes, spatially distant from regions that can be penetrated with microelectrodes. This makes it difficult to estimate the amplitude and time course of the receptor current at the site of transduction and difficult to tell whether conduction along the dendrite occurs actively or passively or how much the receptor current is attenuated by the dendrite if conduction is passive.

In the Pacinian corpuscle, action potentials are assumed to arise at the first node of Ranvier within the corpuscle (Diamond et al., 1956; Loewenstein, 1971), but there is also evidence that voltage-activated sodium channels, and regenerative activity, occur earlier in the sensory neurite (Pawson and Bolanowski, 2002). Action potentials are also thought to start at the first node of Ranvier in muscle spindles, but there is evidence of regenerative processes in the sensory dendrites (Querfurth, 1985). In crustacean stretch receptors, transduction occurs at dendrite tips, electrically distant from the soma, but there is evidence of active currents in the dendrites that contribute to the receptor potential (Swerup and Rydqvist, 1992).

The mechanoreceptor used here is a member of the arthropod

Received Feb. 20, 2003; revised March 1, 2003; accepted May 9, 2003

This work was supported by a grant from the Canadian Institutes of Health Research to A.S.F. We thank Päivi Torkkeli, Ulli Höger, and Alexandre Widmer for advice and help at every stage. Shannon Meisner maintained and bred the animals, as well as provided expert technical help throughout.

Correspondence should be addressed to Dr. Andrew S. French, Department of Physiology and Biophysics, Dalhousie University, Halifax, Nova Scotia, B3H 4H7, Canada. E-mail: andrew.french@dal.ca.

Copyright $\odot 2003$ Society for Neuroscience $\quad$ 0270-6474/03/236096-06\$15.00/0 type I receptors (McIver, 1985; French, 1988), having bipolar sensory neurons with single sensory dendrites that terminate at the presumed sites of mechanotransduction. Previous experiments in insect type I mechanoreceptor neurons using combinations of mechanical and extracellular electrical stimulation and recording suggested that dendrites might be excitable, although action potentials would normally start at the soma (Guillet et al., 1980; Erler and Thurm, 1981). Extracellular experiments in spider type I receptors also suggested that the distal dendrite was excitable (Seyfarth et al., 1982).

The VS-3 slit-sense organ of the spider Cupiennius salei (Barth and Libera, 1970) possesses relatively large bipolar neurons that can be impaled by microelectrodes to allow current-clamp and voltage-clamp recording during mechanical stimulation (French et al., 2002). Immunohistochemical studies indicated that voltage-activated sodium channels are present throughout the sensory dendrites of these neurons, at densities comparable with those in the axon (Seyfarth et al., 1995). Although the somata can produce overshooting action potentials (Torkkeli et al., 2001), frequency-response measurements of VS-3 neurons showed that the delay between stimulation and action potential response was $\sim 0.5$ msec less for mechanically than electrically stimulated neurons, suggesting that action potentials are initiated closer to the site of mechanotransduction in the distal dendrite than to the soma (French et al., 2001).

Here we performed three types of experiments on VS-3 neurons: (1) voltage-clamp measurements of charge recovered during step mechanical stimuli combined with voltage jumps at the soma, (2) current-clamp receptor potential frequency-response measurements during mechanical stimulation, and (3) current- 


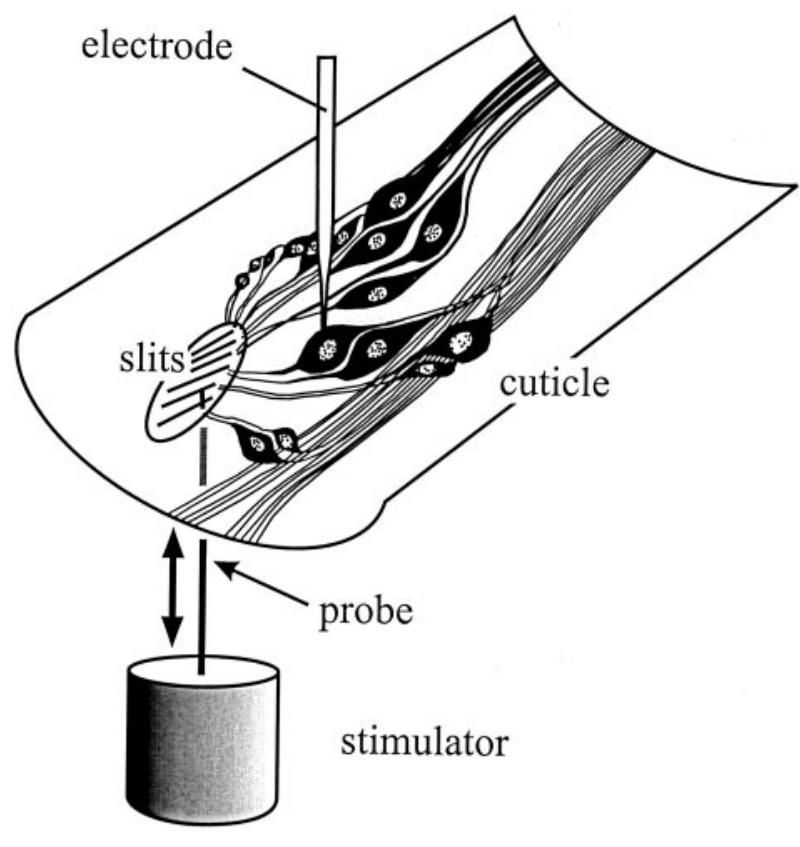

Figure 1. Recording and stimulating VS-3 neurons. A concave piece of patellar cuticle containing the slits and associated neurons was dissected and mounted in a fixed holder. Slits were displaced by a glass probe driven by a piezoelectric stimulator. Voltage-clamp and currentclamp recording and stimulation were performed by the switching single-electrode techniques via an intracellular glass microelectrode lowered from above to penetrate individual neurons.

clamp recordings of active and passive somatic responses to step mechanical stimuli. These data show that earlier measurements underestimated receptor current amplitude at the end of the dendrite and overestimated dendrite space constant, and that action potentials normally arise in the sensory dendrite, close to the site of mechanotransduction.

\section{Materials and Methods}

Preparation. Spiders (C. salei) were taken from a laboratory stock. Legs of adult females were autotomized and dissected under spider saline (Höger et al., 1997) containing (in mM): $223 \mathrm{NaCl}, 6.8 \mathrm{KCl}, 8 \mathrm{CaCl}_{2}, 5.1 \mathrm{MgCl}_{2}$, 10 HEPES, pH 8.0. Legs were split lengthwise, and muscles in the anterior concave sections of the femur, patella, and tibia were removed. Preparations with the intact lyriform slit-sense organ VS-3 [nomenclature of Barth and Libera (1970)] in the patella section were mounted with dental wax onto a custom-designed Plexiglas holder (Fig. 1). Details of the preparation and its dissection have been described previously (Seyfarth and French, 1994; Juusola et al., 1994).

Recording and stimulation. The experimental arrangement was mounted on a gas-driven vibration isolation table (Technical Manufacturing, Peabody, MA) inside a Faraday cage. Using a stereomicroscope, VS-3 receptor cells were identified through a thin layer of saline. A horizontal puller (P-2000; Sutter Instruments, Novato, CA) was used to pull microelectrodes from borosilicate glass ( $1 \mathrm{~mm}$ outer diameter, $0.5 \mathrm{~mm}$ inner diameter). Electrodes ( $3 \mathrm{M} \mathrm{KCl}, 45-70 \mathrm{M} \Omega$ ) were positioned with a micromanipulator (Leitz, Wetzlar, Germany) above a neuronal soma. Cell penetration was achieved by gentle tapping of the micromanipulator. All electrical recordings were done with the switching singleelectrode technique in either current- or voltage-clamp mode [duty cycle, 1:8 stimulating/recording; switching frequency, $20 \mathrm{kHz}$; SEC-05LX amplifier (NPI Electronic, Tamm, Germany)]. Details of the recording technique have been given by Torkkeli and French (1994).

For mechanical stimulation a piezoelectric stimulator (LVPZT, Polytec Physik-Instrumente, Waldbronn, Germany) pushed a glass probe against the cuticular slits from below. Slit deflection of $<2 \mu \mathrm{m}$ was usually sufficient to evoke action potentials in sensory neurons. All experiments were performed at room temperature $\left(22 \pm 2^{\circ} \mathrm{C}\right)$.
Experiments were controlled by an IBM-compatible computer, using custom-written software via 16-bit analog-to-digital and 12-bit digitalto-analog converters (6035E, National Instruments, Austin, TX). The computer provided the mechanical or electrical stimulation and recorded membrane current, membrane voltage, and stimulator deflection with sampling rates of up to $10 \mathrm{kHz}$. Current and voltage signals were low-pass filtered by the voltage-clamp amplifier before digitization (current, $3.3 \mathrm{kHz}$; voltage, $33.3 \mathrm{kHz}$ ).

After cell penetration and the establishment of mechanical stimulation (i.e., mechanically evoked action potentials), the saline was supplemented with $1 \mu \mathrm{M}$ tetrodotoxin (TTX) to block voltage-activated sodium channels and prevent action potentials. All chemicals were purchased from Sigma (Oakville, Ontario), and solutions were prepared fresh or from frozen stock.

Voltage-jump measurements. The voltage-jump technique (Häusser and Roth, 1997) is based on the idea that current flowing through ion channels at the end of a dendrite varies with the intracellular potential at the specific location of the channels. Originally developed to examine synaptic channels, the method is equally applicable to the present situation, where channels at the end of a sensory dendrite are opened by mechanical stimulation. A voltage jump at the soma causes potential change to propagate decrementally outward along the dendrite, so that the time course of current flowing through the sensory channels reflects two interacting processes: voltage propagation along the dendrite and the time-dependent behavior of the channels themselves. Analytical predictions and simulations show that total charge recovered at the soma varies with delay between voltage jump and a step stimulus to give approximately sigmoidal plots, with the two regions before and after the transition reflecting primarily the two time dependent processes (dendritic cable propagation and stimulated ion channels). These plots can then be used to estimate parameters defining the two processes.

Voltage-jump experiments were controlled by the computer via two 12-bit digital-to-analog converters driving both the amplifier and the piezoelectric stimulator. The computer generated a series of steps in position (mechanical steps) and membrane potential (voltage jumps) with varying time separations between the two steps. Membrane current and stimulator position were sampled at $0.1 \mathrm{msec}$ intervals using 16-bit analog-to-digital converters, and total charge produced by the mechanical step was obtained by digitally integrating the receptor current. Charge, $Q$, versus time separation between the mechanical and voltage steps was fitted by:

$$
Q=Q_{0}+\alpha^{2} V_{c} g(-s)\left(1-\mathrm{e}^{-s / \tau}\right)
$$

where $Q_{0}$ is the charge recovered during a mechanical stimulus at the resting potential, $\alpha$ is an attenuation parameter for voltage spread along the dendrite, $V_{c}$ is the change in membrane potential during the voltage step, $g(-s)$ describes the change in conductance during the mechanical stimulus as a function of a time variable, $s$, and $\tau$ is the membrane time constant of the sensory dendrite (Häusser and Roth, 1997). Voltageclamp data were averaged to reduce experimental noise whenever possible. All of the data presented here were obtained from single trials or from two or three averages.

Current-clamp measurements. Action potential and receptor potential responses to mechanical steps were obtained under current clamp using a computer-generated mechanical stimulation and voltage recording protocol with a time resolution of $0.1 \mathrm{msec}$. For receptor potential frequency response measurements, pseudorandom Gaussian white noise was generated by the computer via a 33-bit binary sequence algorithm driving a 12-bit digital-to-analog converter into the piezoelectric stimulator. The position signal from the displacement transducer of the stimulator, and the cell membrane potential, were digitized via a 16-bit analog-to-digital converter and sampled at $1 \mathrm{msec}$ intervals. The bandwidth of the displacement signal was determined by the stimulator circuitry, and the input noise amplitude was below $1 \%$ of its low-frequency value by the sampling Nyquist frequency of $500 \mathrm{~Hz}$. Sampled signals were transferred to the frequency domain using the fast Fourier transform (Cooley and Tukey, 1965) in segments of 512 sample pairs. Frequencyresponse functions (gain and phase) between the mechanical stimulus 
Table 1. Voltage-jump measurements

\begin{tabular}{llll}
\hline Mechanical step amplitude $(\mu \mathrm{m})$ & $Q_{0}(\mathrm{pC})$ & $\alpha$ & $\tau$ (msec) \\
\hline 7 & 3.60 & 1.21 & 5.19 \\
3 & 3.11 & 1.13 & 4.21 \\
5 & 4.64 & 1.41 & 5.18 \\
5 & 3.53 & 1.27 & 7.97 \\
4 & 1.89 & 1.22 & 8.12 \\
3 & 4.32 & 0.77 & 7.25 \\
3 & 1.19 & 0.71 & 6.12 \\
3 & 3.10 & 1.19 & 5.93 \\
Mean \pm SD & $3.17 \pm 1.15$ & $1.11 \pm 0.25$ & $6.25 \pm 1.41$
\end{tabular}

Parameters of Equation 1 after fitting data from eight voltage-jump experiments. Mechanical step duration was 10 msec. All experiments used a voltage jump of $-20 \mathrm{mV}$ from resting potential.

and receptor potential were calculated by direct spectral estimation and plotted as Bode plots of phase and log gain versus log frequency. Coherence functions (Bendat and Piersol, 1980) were calculated from the same data. Frequency-response functions were fitted by a coherence-weighted minimum square error process to the function:

$$
G(j \omega)=\frac{\beta}{\cosh \left(\frac{x}{\lambda} \sqrt{j \omega \tau+1}\right)},
$$

where $G(j \omega)$ is complex gain (containing both amplitude and phase data), as a function of radial frequency, $\omega, j=V-1, \lambda$ is cable space constant, $\tau$ is cable time constant, and $x$ is distance along the cable (Jack et al., 1983). The scaling parameter $\beta$ represents the conversion of mechanical displacement to receptor potential during transduction.

Dendrite length measurements. After electrical recording, preparations were transferred to a compound microscope (BH2, Olympus, Tokyo, Japan) equipped with a digital camera (Axiocam Color, Carl Zeiss, Oberkochen, Germany), and the length of the sensory dendrite from its origin at the soma to its termination in the slit was estimated using image analysis software (AxioVision 3.1, Carl Zeiss). Some preparations were also stained using the fluorescent dye di-8-ANEPPQ (Molecular Probes, Eugene, OR) at $1 \mathrm{~mm}$ concentration for $60 \mathrm{~min}$ and then viewed with an inverted compound microscope (Axiovert 100, Carl Zeiss) and photographed with the same digital camera.

\section{Results}

Data were recorded from 19 VS-3 neurons. After initial recordings of action potentials were made, preparations were treated continuously with TTX (see Materials and Methods) and were either clamped at the resting potential $($ mean $=-62.9 \pm 9.3 \mathrm{mV})$ during voltage-clamp experiments or allowed to remain at the resting potential during current-clamp experiments. Under these conditions, the known voltage-activated sodium, calcium, and potassium currents would not be active (Sekizawa et al., 1999, 2000; Torkkeli et al., 2001).

\section{Voltage-jump experiments}

Voltage-clamped VS-3 neurons were stimulated with mechanical steps of 10 msec duration and amplitudes in the range of 3 to 7 $\mu \mathrm{m}$ (Table 1). Membrane potential was stepped by $-20 \mathrm{mV}$ (hyperpolarizing voltage jump) for $200 \mathrm{msec}$ with a variable delay between the voltage and mechanical steps. The delay was usually varied from $-10 \mathrm{msec}$ (voltage jump before mechanical step) to $30 \mathrm{msec}$ (voltage jump after mechanical step). Total charge recovered at the soma was estimated by integrating the recorded current during the mechanical step and for the following $5 \mathrm{msec}$. Current was measured relative to a baseline value established during $50 \mathrm{msec}$ before the steps. Mechanically induced current increased with hyperpolarization because of the increased driving force across the transduction channels relative to the equilibrium

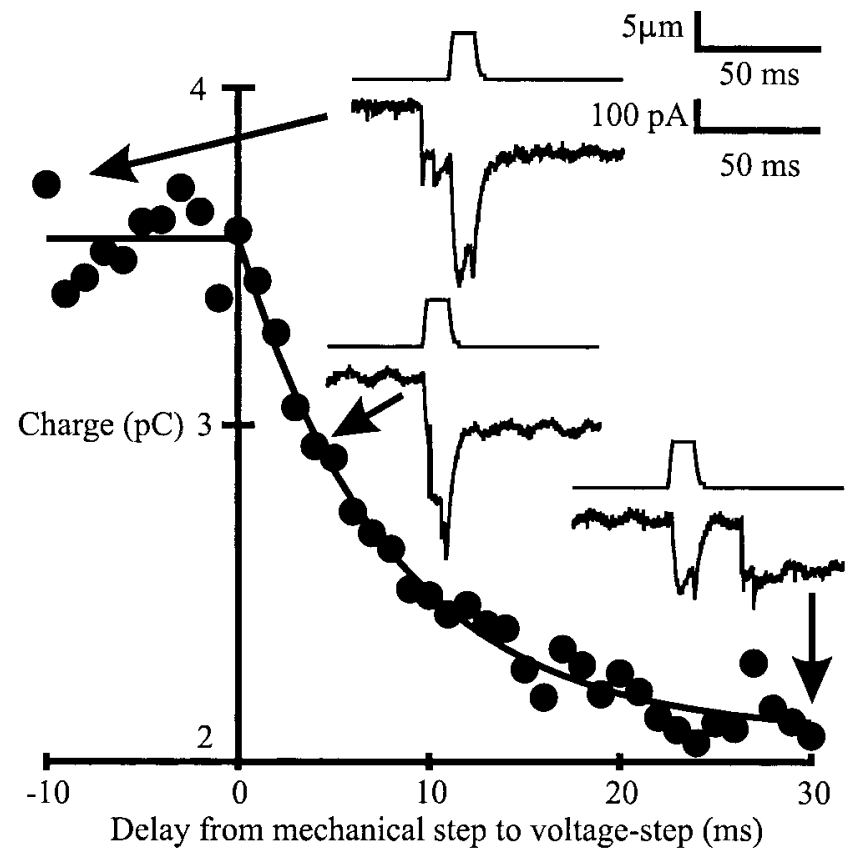

Figure 2. Sensory dendrite voltage-jump experiment. A VS-3 neuron was clamped at its resting potential $(-69 \mathrm{mV})$ and stimulated with a $5 \mu \mathrm{m}$ step mechanical deflection of $10 \mathrm{msec}$ duration. The membrane potential was stepped by $-20 \mathrm{mV}$ (hyperpolarizing) at varying times relative to the mechanical step. The main part of the figure shows recovered charge in the soma caused by single mechanical steps as a function of delay between the mechanical and voltage steps, fitted with Equation 1 ( $Q_{0}=3.53 \mathrm{pC}, \alpha=1.27, \tau=7.97 \mathrm{msec}$ ). Insets show the actual recordings of mechanical steps from the position transducer and resulting current records at delays of $-10,4$, and $30 \mathrm{msec}$, corresponding to the indicated points on the main figure.

potential of $\mathrm{Na}^{+}$, the dominant permeant ion (Höger et al., 1997), so the recovered charge was greatest when the voltage jump preceded the mechanical step (Fig. 2).

The voltage-jump technique was designed originally to estimate synaptic current at the end of dendritic cable processes (Häusser and Roth, 1997) but is equally applicable to the present situation in which the current was produced by sensory transduction. Plots of recovered charge versus delay between the voltage jump and the mechanical stimulus have been shown by simulation and analytical considerations to reflect two processes, the time course of the conductance change (synaptic or receptor current) and the spread of the voltage jump along the dendritic cable (Häusser and Roth, 1997).

Equation 1 includes a term, $g(-s)$, that represents conductance as a function of time produced by the mechanical step. The asymptotic conductance of VS-3 receptor channels at large negative potentials was previously estimated to be $4.6 \pm 1.5 \mathrm{nS}$ (Höger et al., 1997), and this is in reasonable agreement with recent estimates by noise analysis of $\sim 500$ mechanoreceptor channels with a single-channel conductance of $7 \mathrm{pS}$ (Höger and French, 1999a, 2002). Therefore, we initially assumed that $g(-s)$ was a $\delta$ function (an infinitely narrow pulse with finite area) of area $4.6 \mathrm{nS}$ multiplied by mechanical step duration $(10 \mathrm{msec})$. Recovered charge versus delay between voltage and mechanical steps was well fitted by Equation 1 using the $\delta$ function assumption (Fig. 2). Mean membrane time constant was $6.25 \mathrm{msec}$, comparable with the value of $\sim 7.0 \mathrm{msec}$ obtained in intact VS-3 neurons (Juusola and French, 1998) but somewhat lower than estimates of $\sim 10 \mathrm{msec}$ obtained in hypodermis preparations of VS-3 organs with crushed axons and dendrites (Sekizawa et al., 1999). 


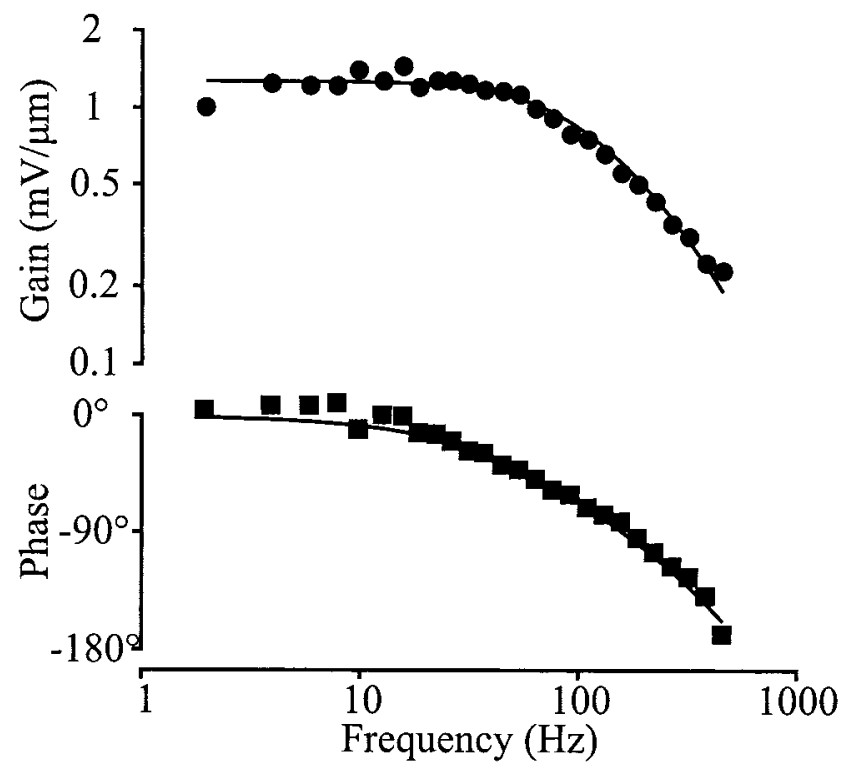

Figure 3. Sensory dendrite frequency-response. Gain and phase components of the complex frequency-response function were obtained by stimulating a VS-3 neuron with pseudorandom white noise displacement of the slit ( $0.397 \mu \mathrm{m}$ rms amplitude) while recording the resultant receptor potential in the soma under current clamp. Solid lines were obtained by fitting with Equation $2(x / \lambda=1.03, \beta=1.92 \mathrm{mV} / \mu \mathrm{m}, \tau=6.25 \mathrm{msec}$ ).

\section{Dendrite frequency-response functions}

Current-clamped VS-3 neurons were stimulated with Gaussian distributed random white noise mechanical deformation of the slits while the resultant fluctuations in membrane potential were recorded. Frequency-response functions for mechanotransduction and conduction along the sensory dendrite (Fig. 3) were well fitted by Equation 2. However, the relatively mild low-pass characteristic of the system did not allow reliable separation of all three parameters $(x / \lambda, \beta$, and $\tau)$, so the value of $\tau$ was fixed at 6.25 msec, on the basis of the voltage-jump experiments (Table 1) and previous estimates (Juusola and French, 1998). Actual dendrite lengths, $x$, were measured by photomicroscopy (Fig. 4) to allow estimation of dendrite space constant, $\lambda$, from $x / \lambda$. Table 2 summarizes the results of frequency-response measurements and cable equation fitting for five VS-3 neurons.

\section{Passive and active responses to mechanical steps}

Experiments were performed to measure the timing of the receptor potential and action potential waveforms relative to the stimulator position during step mechanical displacements. The rapid upswing of the action potentials always occurred early in the rising phase of the position signal (Fig. 5). In contrast, after TTX application, the rising phase of the receptor potential was always delayed by at least $1 \mathrm{msec}$ (Fig. 5). Similar results were obtained from five separate experiments on different preparations.

\section{Discussion}

Transduction occurs at the ends of sensory dendrites in many sensory receptors of vertebrates and invertebrates, but little is known about the electrical properties of these dendrites or their roles in sensory processing. In the analogous situation of CNS dendrites, the diverse expression of ion channels causes a wide range of excitability and electrical signal processing (Häusser et al., 2000), so similar functional variety may occur in sensory dendrites. The present experiments were designed to measure the passive membrane properties of the sensory dendrites in spider

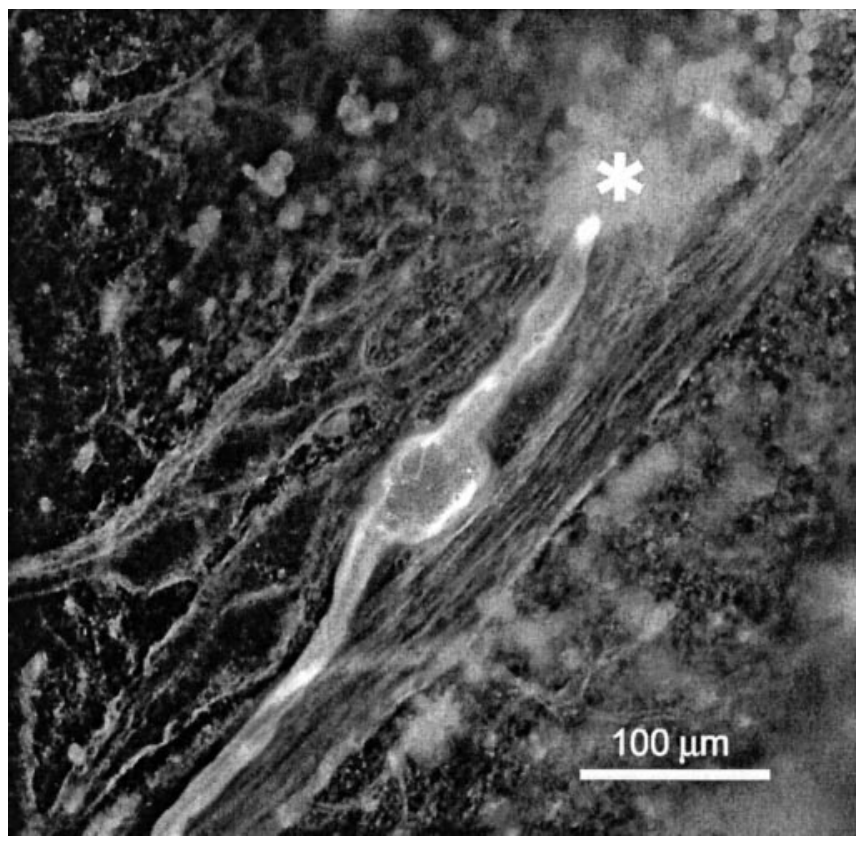

Figure 4. Sensory dendrite length. Photomicrograph of VS-3 neurons stained with di-8ANEPPQ. The neuron used for the experiment shown in Figure 3 was identified visually during the experiment, and its outline was enhanced digitally using imaging software (Adobe Photoshop 7.0) for easy identification. Dendrite lengths, $x$, from tips to junctions with the somata were measured from similar photomicrographs using AxioVision software.

Table 2. Dendrite frequency-response functions

\begin{tabular}{lllll}
\hline Stimulus amplitude $(\mu \mathrm{m} \mathrm{rms})$ & $x / \lambda$ & $x(\mu \mathrm{m})$ & $\lambda(\mu \mathrm{m})$ & $\beta(\mathrm{mV} / \mu \mathrm{m})$ \\
\hline 0.472 & 0.69 & 200 & 289 & 3.89 \\
0.260 & 0.92 & 280 & 304 & 2.54 \\
0.505 & 0.82 & 110 & 134 & 3.34 \\
0.397 & 1.03 & 120 & 114 & 1.92 \\
0.401 & 0.95 & 200 & 211 & 1.72 \\
Mean \pm s.d. & $0.99 \pm 0.14$ & $182 \pm 69.4$ & $208 \pm 89.5$ & $2.68 \pm 0.92$ \\
\hline
\end{tabular}

Parameters of Equation 2 obtained by fitting frequency-response functions from five VS-3 neurons. Dendrite lengths, $X$, were measured by microscopy (Fig. 4). Membrane time constant, $\tau$, was fixed at $6.25 \mathrm{msec}$ in all experiments. At least 50 sec of data was processed for each cell.

VS-3 neurons and to test the hypothesis that action potentials are initiated in the neuronal somata after passive propagation of the receptor current along the dendrite.

The voltage-jump and receptor-potential frequency responses measured passive dendrite properties as signals spread in opposite directions along the dendrite, voltage-jump measuring propagation of voltage change from the soma to sensory channels at the dendrite tip, and frequency responses measuring propagation of receptor potential to the soma. These measurements were independent of each other, except for membrane time constant, which was based on voltage-jump experiments and in good agreement with previous estimates from these neurons (Juusola and French, 1998).

\section{Time course of the receptor current}

Voltage-jump plots reflect the time courses of two processes: the current source at the distal end of the dendrite and passive dendritic cable propagation (Häusser and Roth, 1997). In our experiments, the time course of receptor current after a step movement would be expected to dominate the trace at negative times (Fig. 2, left of zero), whereas the time course of dendritic propagation would dominate the trace at positive times. The sharp transition 


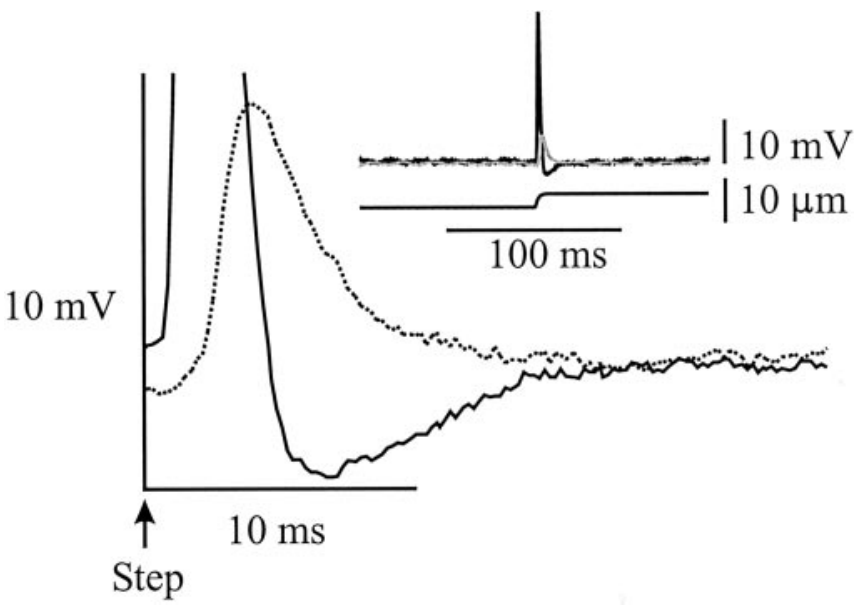

Figure 5. Current-clamp response timing. Membrane potential responses in the soma of a VS-3 neuron to a $3 \mu \mathrm{m}$ step displacement of the slit. The main figure shows superimposed current-clamp recordings of membrane potential before (solid line) and after (dashed line) application of TTX during the first $25 \mathrm{msec}$ after the mechanical step. The complete recordings, together with the position transducer output below, are shown in the inset on a longer time scale. No change in membrane potential occurred after application of TTX, but the action potential was suppressed, leaving a receptor potential of $\sim 5 \mathrm{mV}$ amplitude. Note that the action potential commenced during the rising phase of the movement, whereas the receptor potential rose $>1$ msec later.

at time 0 , and the good fit by Equation 1, indicates that most of the receptor current after a step movement is a rapidly decaying transient impulse, much faster than the dendritic cable time constant, $\tau=6.25 \mathrm{msec}$, which is comparable with the previous estimate of $7.0 \mathrm{msec}$ obtained in the same preparation (Juusola and French, 1998). There must also be a smaller, slower component of receptor current with a time constant of $>100 \mathrm{msec}$, based on previous voltage clamp measurements (Höger and French, 1999a), but these earlier measurements also indicated that there is a large transient component.

\section{Conductance of the receptor channels}

Parameter $Q_{0}$ in Equation 1 measured the total charge produced by a step movement when the membrane potential was $-20 \mathrm{mV}$ below the resting potential (approximately $-85 \mathrm{mV}$ ). The mean value of $3.17 \mathrm{pC}$ would correspond to a current of $317 \mathrm{pA}$ flowing through the transduction channels if the current were constant during the $10 \mathrm{msec}$ step, which agrees with earlier measurements of receptor current under voltage clamp at hyperpolarized potentials (Höger et al., 1997). However, this apparent agreement is probably coincidental because a large part of the current seems to be transient.

The attenuation parameter, $\alpha$, describes the amount by which the voltage jump in the soma is reduced by cable conduction along the dendrite. The mean value of 1.11 cannot be correct, because it must be less than unity. In fact, the frequency-response data suggest a value of $\sim 0.37$. The most reasonable explanation for this discrepancy is that our estimate of the conductance parameter, $g$, was too small. We used a value of $4.6 \mathrm{nS}$, based on peak current-voltage measurements that assumed perfect voltage clamp of the distal dendrite, although it was recognized at the time that significant deviation might occur because of the dendrite cable (Höger et al., 1997). It now seems likely that we underestimated the total conductance of the receptor channels by a factor of at least 3 , so a value of $\sim 15 \mathrm{nS}$ would be indicated.

Estimates of single-receptor channel conductance and number of receptor channels in this preparation have been made pre- viously by noise analysis (Höger and French, 1999a; 2002). The most recent measurements gave values of $\sim 7 \mathrm{pS}$ for singlechannel conductance and 500 channels per cell, for a total conductance of $\sim 3.5 \mathrm{nS}$. Although these estimates were based on voltage-clamp data, they were conducted at the resting potential, reducing potential errors in the clamp at the dendrite tips. However, they used saturating mechanical steps, so the initial receptor current may have significantly depolarized the dendrite tips, reducing the receptor current and the estimate of single-channel conductance. Another possible source of error was the estimated equilibrium potential of the permeant ion, $\mathrm{Na}^{+}$, which was based on estimates of $\mathrm{Na}^{+}$concentrations both inside and outside the cell that have not been verified for accuracy. It seems likely that the single-channel conductance of VS-3 receptor channels is significantly higher than earlier estimates and has at least two components with transient and slow time courses. Whether these represent complex kinetic properties of one class of ion channels, or the existence of more than one type of mechanically activated channel, remains unclear.

\section{Dendrite cable properties}

Earlier estimates of VS-3 dendrite cable properties were based on measured electrical parameters of the somatic membrane and general estimates of intracellular resistivity (Höger et al., 1997). The previous estimate of $600 \mu \mathrm{m}$ for the dendrite length constant is now seen to be much too large. This error could be attributable to incorrect values of the electrical properties or the diameters of the dendrites. Of these, the latter are probably the most difficult to measure accurately in living tissue and could easily have been overestimated. The membrane time constant value of $6.25 \mathrm{msec}$ obtained here for the dendrite is lower than previous estimates in the soma (Juusola and French, 1998) and significantly lower than estimates of $\sim 10 \mathrm{msec}$ in VS-3 neurons with crushed axons and dendrites (Sekizawa et al., 1999). This might reflect the existence of more open ion channels in the dendrite membrane than the soma, or reduced dendrite membrane capacity, because the dendrites are extensively wrapped by glial cells (Seyfarth et al., 1995).

\section{Action potential initiation}

Extracellular measurements in both insects (Guillet et al., 1980; Erler and Thurm, 1981) and spiders (Seyfarth et al., 1982) have indicated that the sensory dendrites of cuticular mechanoreceptors might be electrically excitable, although this has never been observed directly. We previously found evidence that the dendrites of VS-3 neurons have a similar density of voltage-activated $\mathrm{Na}^{+}$channels to the axons, supporting this idea (Seyfarth et al., 1995). The present data add further support, because the length constant of the dendrite would significantly attenuate a passively conducted receptor current, and action potentials always arrive in the soma before the receptor potential (Fig. 5). This latter finding, of $\sim 1$ msec delay between action potentials and receptor potentials, is in good agreement with our earlier frequency-response measurements (French et al., 2001), which found that mechanical stimulation could produce action potentials at least $0.5 \mathrm{msec}$ faster than electrical stimulation. This surprising finding could be the result of several factors, including differing membrane capacitances and ion channel densities in the soma and dendrite, as discussed above.

Combination of the transduction parameter, $\beta=2.68 \mathrm{mV} /$ $\mu \mathrm{m}$, with the measured voltage threshold for action potential production in the soma of $\sim 30 \mathrm{mV}$ (Sekizawa et al., 1999) suggests a slit displacement threshold of $\sim 10 \mu \mathrm{m}$, whereas the actual mechanical threshold can be as low as $2 \mu \mathrm{m}$ (Höger et al., 1997). 
This indicates that the site of action potential initiation in the dendrite tip has a significantly lower voltage threshold than the soma, as might be expected.

The current-clamp measurements suggest that action potentials arrive in the soma $<1 \mathrm{msec}$ after the start of a step movement (Fig. 5). Because the dendrites are $\sim 200 \mu \mathrm{m}$ long, action potentials initiated at the distal end of the dendrite would require a conduction velocity of at least $0.2 \mathrm{~m} / \mathrm{sec}$ to reach the soma in this time. Little is known about conduction velocities in arthropod dendrites. Chapman and Pankhurst (1967) measured conduction velocities in a range of cockroach axons and found an approximately square root relationship between velocity and fiber diameter Extrapolation of their data to VS-3 dendrites of 1-2 $\mu \mathrm{m}$ diameter (Seyfarth et al., 1995) would predict a conduction velocity of at least $1 \mathrm{~m} / \mathrm{sec}$. Höger and French (1999b) found a mean conduction velocity of $5 \mathrm{~m} / \mathrm{sec}$ for VS-3 axons with diameters of $5-10 \mu \mathrm{m}$, which is faster than predicted by the cockroach data, so a minimum conduction velocity of $0.2 \mathrm{~m} / \mathrm{sec}$ in the dendrites seems reasonable. The VS-3 organ is $\sim 3 \mathrm{~cm}$ from the cephalothorax in an adult animal, so an additional conduction delay of 5-10 msec can be expected before the information arrives in the CNS.

\section{Conclusions}

The data presented here indicate that the sensory dendrites of the VS-3 neurons would significantly attenuate and retard the receptor current if it flowed passively from mechanically activated ion channels at the dendrite tips to an initiation site in the somata. They also support the idea that action potentials normally arise at or near a low threshold region in the dendrite tips and propagate actively along the dendrites to the somata. This would provide the most rapid detection of mechanical events and facilitate the perception of rapidly changing mechanical signals such as vibrations. It seems likely that this situation is common to other arthropod mechanoreceptors and possibly to arthropod thermoreceptors and chemoreceptors that have similar morphology. Recent evidence indicates that mammalian mechanoreceptors may also have active sensory endings (Pawson and Bolanowski, 2002). Active conduction from the site of mechanotransduction would allow the most rapid detection of sensory stimuli, which has obvious evolutionary advantages, and seems likely to occur in many vertebrate and invertebrate sensory receptors.

\section{References}

Barth FG, Libera W (1970) Ein Atlas der Spaltsinnesorgane von Cupiennius salei Keys. Chelicerata (Araneae). Z Morph Tiere 68:343-369.

Bendat JS, Piersol AG (1980) Engineering applications of correlation and spectral analysis. New York: Wiley.

Chapman KM, Pankhurst JH (1967) Conduction velocities and their temperature coefficients in sensory nerve fibres of cockroach legs. J Exp Biol 46:63-84

Cooley JW, Tukey JW (1965) An algorithm for the machine calculation of complex Fourier series. Math Comput 19:297-301.

Diamond J, Gray JAB, Sato M (1956) The site of initiation of impulses in Pacinian corpuscles. J Physiol (Lond) 113:54-67.

Erler G, Thurm U (1981) Dendritic impulse initiation in an epithelial sensory neuron. J Comp Physiol 142:237-249.

French AS (1988) Transduction mechanisms of mechanosensilla. Annu Rev Entomol 33:39-58.

French AS, Höger U, Sekizawa S-i, Torkkeli PH (2001) Frequency response functions and information capacities of paired spider mechanoreceptor neurons. Biol Cybern 85:293-300.

French AS, Torkkeli PH, Seyfarth EA (2002) From stress and strain to spikes: mechanotransduction in spider slit sensilla. J Comp Physiol [A] 188:739-752.

Guillet JC, Bernard J, Coillot JP, Callec JJ (1980) Electrical properties of the dendrite in an insect mechanoreceptor: effects of antidromic or direct electrical stimulation. J Insect Physiol 26:755-762.

Häusser M, Roth A (1997) Estimating the time course of the excitatory synaptic conductance in neocortical pyramidal cells using a novel voltage jump method. J Neurosci 17:7606-7625.

Häusser M, Spruston N, Stuart GJ (2000) Diversity and dynamics of dendritic signaling. Science 290:739-744.

Höger U, French AS (1999a) Estimated single-channel conductance of mechanically-activated channels in a spider mechanoreceptor. Brain Res $826: 230-235$.

Höger U, French AS (1999b) Temperature sensitivity of transduction and action potential conduction in a spider mechanoreceptor. Pflügers Arch 438:837-842.

Höger U, French AS (2002) Extracellular acid increases the open probability of transduction channels in spider mechanoreceptors. Eur J Neurosci 16:2311-2316.

Höger U, Torkkeli PH, Seyfarth E-A, French AS (1997) Ionic selectivity of mechanically activated channels in spider mechanoreceptor neurons. J Neurophysiol 78:2079-2085.

Jack JJB, Noble D, Tsien RW (1983) Electric current flow in excitable cells. Oxford: Clarendon.

Juusola M, French AS (1998) Adaptation properties of two types of sensory neurons in a spider mechanoreceptor organ. J Neurophysiol 80:2781-2784.

Juusola M, Seyfarth E-A, French AS (1994) Sodium-dependent receptor current in a new mechanoreceptor preparation. J Neurophysiol 72:3026-3028.

Loewenstein WR (1971) Mechano-electric transduction in the Pacinian corpuscle: initiation of sensory impulses in mechanoreceptors. Handbook Sensory Physiol 1:269-290.

McIver SB (1985) Mechanoreception. In: Comprehensive insect physiology biochemistry and pharmacology (Kerkut GA, Gilbert LI, eds), pp 71-132. Oxford: Pergamon.

Pawson L, Bolanowski SJ (2002) Voltage-gated sodium channels are present on both the neural and capsular structures of Pacinian corpuscles. Somatosens Mot Res 19:231-237.

Querfurth H (1985) Receptor potentials of isolated frog muscle spindle evoked by sinsusoidal stimulation. J Neurophysiol 53:60-75.

Sekizawa S-i, French AS, Höger U, Torkkeli PH (1999) Voltage-activated potassium outward currents in two types of spider mechanoreceptor neurons. J Neurophysiol 81:2937-2944.

Sekizawa S-i, French AS, Torkkeli PH (2000) Low-voltage-activated calcium current does not regulate the firing behavior in paired mechanosensory neurons with different adaptation properties. J Neurophysiol 83:746-753.

Seyfarth E-A, French AS (1994) Intracellular characterization of identified sensory cells in a new spider mechanoreceptor preparation. J Neurophysiol 71:1422-1427.

Seyfarth E-A, Bohnenberger J, Thorson J (1982) Electrical and mechanical stimulation of a spider slit sensillum: outward current excites. J Comp Physiol [A] 147:423-432.

Seyfarth E-A, Sanders EJ, French AS (1995) Sodium channel distribution in a spider mechanosensory organ. Brain Res 683:93-101.

Swerup C, Rydqvist B (1992) The abdominal stretch receptor organ of the crayfish. Comp Biochem Physiol [A] 103:423-431.

Torkkeli PH, French AS (1994) Characterization of a transient outward current in a rapidly adapting insect mechanoreceptor neuron. Pflügers Arch 429:72-78.

Torkkeli PH, Sekizawa S-i, French AS (2001) Inactivation of voltageactivated $\mathrm{Na}^{+}$currents contributes to different adaptation properties of paired mechanosensory neurons. J Neurophysiol 85:1595-1602. 